\title{
Comprehensive Emergency Obstetrical and Neonatal Care (CEmONC) at Karnali Academy of Health Sciences, Teaching Hospital, Jumla
}

\author{
Gautam SK ${ }^{1}$, Dahal M² \\ ${ }^{1}$ Associate Professor, School of Nursing and Midwifery, Karnali Academy of Health Science (KAHS), Jumla, \\ Nepal \\ ${ }^{2}$ Nursing officer, School of Nursing and Midwifery, KAHS, Jumla, Nepal
}

Corresponding Author:

Saraswoti Kumari Gautam Bhattarai

Email: saraswotigautam@gmail.com

\begin{abstract}
Introduction: Providers skilled in emergency obstetric and newborn care (EmONC) services are essential, particularly in countries like Nepal with a high burden of maternal and newborn mortality. So this study aims to find out the status of comprehensive emergency obstetrical and neonatal care (CEmONC) service.

Method: A retrospective cross-sectional study was conducted using secondary data sources at maternity ward of KAHS teaching hospital, Jumla. Total 291 women admitted in maternity ward for childbirth were included in the study of six month period of 2075. Sampling technique was census for the study who were admitted for child birth purpose. Cases were selected from the record of the maternity ward. The data was collected by using structured tool. Ethical approval was taken from the ethical review committee of KAHS for ethical clearance. Data was analyzed by using descriptive statistics.

Result: There were 291 women admitted in the maternity ward for the purpose of childbirth during six-month period. Among them 224(76.97\%) women delivered baby by spontaneous vaginal delivery; 61(20.96\%) delivered with $\mathrm{C} / \mathrm{S}$ and 6(2.06\%) were delivered with instrumental delivery. Regarding the indication of 61 cesarean section $(\mathrm{C} / \mathrm{S})$ delivery; $22.95 \%$ with fetal distress, $16.39 \%$ with cephalopelvic disproportion and $11.47 \%$ with meconium stained liquor

Conclusions: About one third childbirth was done by cesarean section with indication of fetal distress, cephalo-pelvic disproportion and meconium stained liquor in higher proportion. Although CEONC service is effective, the rate of cesarean section can be reduced by providing good quality antenatal care.
\end{abstract}

Keywords: Emergency obstetrical care; Newborn care; Spontaneous vaginal delivery 


\section{INTRODUCTION}

Maternal mortality is a measure of a woman's risk of dying during pregnancy, childbirth or during the 42 days following delivery ${ }^{1}$. Approximately $15 \%$ of expected births worldwide will result in life-threatening complications during pregnancy, delivery, or the postpartumperiod ${ }^{2}$. The concept of emergency obstetric and newborn care (EmONC) was introduced by WHO, UNICEF, and UNFPA in 1997 as an organizing framework for the delivery of evidence-based clinical services to reduce maternal and newborn mortality ${ }^{3}$. Skilled birth attendants $(\mathrm{SBAs})^{4}$ provide EmONC services within the context of community-focused and facility-based health systems, enabling timely prevention and intervention for these complications and saving the lives of mothers and newborns. Universal access to EmONC is considered essential to reduce maternal mortality . Pregnancy complications can be unpredictable and many women in developing countries do not have access to health facilities where lifesaving care is available. Emergency obstetric care is one of the strategies to reduce maternal mortality. It refers to lifesaving services for maternal health complications being provided by a skilled health worker either in health facilities or home. High utilization of emergency obstetric care services can reduce maternal deaths and disabilities drastically in most of the developing countries ${ }^{5}$. Emergency obstetric and newborn care is the timely care given to women and newborns experiencing complications during delivery. Comprehensive emergency obstetrical and newborn care (CEONC) includes CS, blood transfusion and neonatal resuscitation in addition to the seven basic signal functions (administration of parenteral antibiotics, uterotonic drugs, and/or parenteral anticonvulsants as needed; manual removal of the placenta; removal of retained products; assisted vaginal delivery; basic neonatal resuscitation) ${ }^{7}$. Therefore, the researcher is interested to identify the status of comprehensive emergency obstetrical and neonatal care services at Karnali academy of health sciences, teaching hospital, Jumla.

\section{METHODS}

A retrospective cross-sectional study was conducted using secondary data sources at maternity ward of KAHS teaching hospital Jumla. Total 291 women admitted in maternity ward for childbirth were included in the study of six month period of 2075 . Sampling technique was census for the study who were admitted for child birth purpose. Cases were selected from the record of the maternity ward. The data was collected by using structured tool. Ethical approval was taken from the ethical review committee of KAHS for ethical clearance. Data was analyzed by using descriptive statistics.

\section{RESULTS}

Table 1: Types of Delivery $(n=291)$

\begin{tabular}{|l|c|c|}
\hline \multicolumn{1}{|c|}{ Mode of delivery } & No. & Percentage \\
\hline Spontaneous vaginal delivery & 224 & 76.97 \\
\hline Cesarean Section & 61 & 20.96 \\
\hline Vacuum & 3 & 1.03 \\
\hline Forceps delivery & 3 & 1.03 \\
\hline
\end{tabular}

Total 291 women came for the purpose of delivery of baby during six-month period. Among them 224 (76.97\%) gave birth by spontaneous vaginal delivery; $61(20.96 \%)$ by cesarean section; $3(1.03 \%)$ by vacuum and $3(1.03 \%)$ by forceps delivery.

Table 2 reveals the indications of Cesarean section (CS). Among 61 cesarean section: 14 (22.95\%) had fetal distress; 10 (16.39\%) cephalopelvic disproportion; 7 (11.47\%) with meconium stained liquor with fetal distress; 5 (8.20\%) previous $\mathrm{C} / \mathrm{S} ; 5$ (8.20\%) prolonged labour; 4 (6.55\%) malpresentation; 3 (4.92\%) eclampsia/severe pre-eclampsia; 3 (4.92\%) failed induction; 3 (4.92\%) transverse lies; 2 (3.27\%) postdated with IUGR; 2 (3.27\%) oligohydramnios; $2(3.27 \%)$ bad obstetric history; 1 (1.64\%) deep transverse arrest. 
Table 2: Indications for Cesarean Section

(CS) Birth $(n=61)$

\begin{tabular}{|l|c|c|}
\hline \multicolumn{1}{|c|}{ Indications } & Number & Percentage \\
\hline Fetal distress & 14 & 22.95 \\
\hline $\begin{array}{l}\text { Cephalo-pelvic } \\
\text { disproportion }\end{array}$ & 10 & 16.39 \\
\hline $\begin{array}{l}\text { Meconium Stained } \\
\text { Liquor(MSL) with fetal } \\
\text { distress }\end{array}$ & 7 & 11.47 \\
\hline Previous CS & 5 & 8.20 \\
\hline Prolonged labour & 5 & 8.20 \\
\hline Malpresentation & 4 & 6.55 \\
\hline $\begin{array}{l}\text { Eclampsia/severe } \\
\text { pre-eclampsia }\end{array}$ & 3 & 4.92 \\
\hline Failed induction & 3 & 4.92 \\
\hline Transverse lie & 3 & 4.92 \\
\hline Postdated with IUGR & 2 & 3.27 \\
\hline Oligohydramnios & 2 & 3.27 \\
\hline Bad obstetric history & 2 & 3.27 \\
\hline Deep transverse arrest & 1 & 1.64 \\
\hline
\end{tabular}

\section{DISCUSSION}

Among 291 women who came for the purpose of delivery of baby during six-month period, 224 (76.97\%) gave birth by spontaneous vaginal delivery; 61 (20.96\%) by cesarean section; 3 (1.03\%) by vacuum and $3(1.03 \%)$ by forceps delivery. One retrospective study done in Ethopia had near similar finding that is $25 \%$ Cesarean delivery ${ }^{8}$.

In regard to indications among 61 Cesarean delivery: $14(22.95 \%)$ had fetal distress whereas a study done in Okhaldhunga $19.8 \%$ had indication of fetal distress 9 Similarly, a study done in Bangladesh had 24\% cases with fetal distress ${ }^{10}$. In this study $10(16.39 \%)$ had cephalopelvic disproportion; 7 (11.47\%) with meconium stained liquor with fetal distress; and 5 (8.20\%) with previous C/S. Likewise $5(8.20 \%)$ with prolonged labour which is contrast

\section{DISCUSSION}

Among 291 women who came for the purpose of delivery of baby during six-month period, 224 (76.97\%) gave birth by spontaneous vaginal delivery; $61(20.96 \%)$ by cesarean section; $3(1.03 \%)$ by vacuum and $3(1.03 \%)$ by forceps delivery. One retrospective study done in Ethopia had near similar finding that is $25 \%$ Cesarean delivery ${ }^{8}$.

In regard to indications among 61 Cesarean delivery: $14(22.95 \%)$ had fetal distress whereas a study done in Okhaldhunga $19.8 \%$ had indication of fetal distress 9. Similarly, a study done in Bangladesh had 24\% cases with fetal distress ${ }^{10}$. In this study $10(16.39 \%)$ had cephalopelvic disproportion; 7 (11.47\%) with meconium stained liquor with fetal distress; and 5 (8.20\%) with previous $\mathrm{C} / \mathrm{S}$. Likewise $5(8.20 \%)$ with prolonged labour which is contrast finding with a study done in Okhaldhunga that is $26.4 \%{ }^{9}$ and a study done in Bangladesh showed $16 \%{ }^{10}$. In this study 4 (6.55\%) $\mathrm{C} / \mathrm{S}$ was done due to malpresentation where as a study done in Okhaldhunga showed $25.3 \%{ }^{9}$. In this study 3 (4.92\%) had indication of eclampsia/ severe pre-eclampsia; $3(4.92 \%)$ failed induction; $3(4.92 \%)$ transverse lie; and $2(3.27 \%)$ postdated with IUGR and a contrast finding was found about postdated on study done in Bangladesh that is $13 \%{ }^{10}$. Similarly, cesarean delivery was done 2 (3.27\%) for oligohydramnios which is contrast finding with a study done in Bangladesh which showed $14 \%{ }^{10}$. Furthermore, $\mathrm{C} / \mathrm{S}$ was done to $2(3.27 \%)$ with bad obstetric history and $1(1.64 \%)$ deep transverse arrest in the study area.

\section{CONCLUSION}

About one third childbirth was done by cesarean section with indication of fetal distress, cephalo-pelvic disproportion and meconium stained liquor in higher proportion. Although CEONC service is effective, the rate of cesarean section can be reduced by providing good quality of antenatal care 


\section{ACKNOWLEDGEMENT}

The authors would like to acknowledge to staffs of Maternity ward and record section of KAHS for the study and to institutional review committee (IRC) for ethical clearance.

\section{REFERENCES}

1. Kassebaum NJ, Bertozzi-villa A, Coggeshall MS, Shackelford KA, Steiner C, Heuton KR, et al. Global, regional, and national levels and causes of maternal mortality during 1990-2013: a systematic analysis for the Global Burden of Disease Study 2013. Lancet. 2014; 384:9801004

2. WHO, UNICEF, UNFPA, World Bank. Trends in maternal mortality: 1990 to 2010 . Geneva: WHO; 2012.

3. Paxton A,Maine D, Freedman L, Fry D, Lobis $\mathrm{S}$. The evidence for emergency obstetric care. Int J GynecolObstet 2005;88(2):181-93.

4. World Health Organization, International Confederation of Midwives, International federation of Gynecology and Obstetrics. Making pregnancy safer: the critical role of the skilled attendant. A joint statement by WHO, ICM and FIGO. Geneva: WHO; 2004

5. Campbell OM, GrahamWJ, Lancet Maternal Survival Series steering group. Strategies for reducing maternal mortality: getting on with what works. Lancet 2006; 368(9543):1284-99

6. Bhandari TR, Dangal G. Emergency Obstetric Care: Strategy for Reducing Maternal Mortality in Developing Countrie:s NJOG 2014 Jan-Jun; $17(1): 8-16$

7. Devkota, M., G. Shakya, N. Pratap K.C., M. Dariang, M. T. Upadhyay, S. Karn, L. Hulton, M. Koblinsky (2011) Readiness of Comprehensive Obstetric and Neonatal Emergency Care in Nepal, National Health Sector Support Programme and Ministry of Health and Population of Nepal, Kathmandu, December 2011.

8. Ayano B, Guto A. Indications and Outcomes of Emergency; Caesarean Section at St Paul's HospitalMedical College, Addis Ababa, Ethiopia 2017: Invest Gynecol Res Women's Health; 2(2) 2015

9. Samdal LJ, Steinsvik KR, Pun P, Dani P, Roald B, Stray-Pedersen B, Bøhler E. The Journal of Obstetrics and Gynecology of India (September-October 2016) 66(S1):S284-S288

10. Begum T, Rahman A, Nababan H, HoqueDM.E, Khan AF, Ali T, et al. (2017) Indications and determinants of caesarean section delivery: Evidence from a population-based study in Matlab, Bangladesh. PLoS ONE 12(11): e0188074. 